\title{
MiniLMv2: Multi-Head Self-Attention Relation Distillation for Compressing Pretrained Transformers
}

\author{
Wenhui Wang Hangbo Bao Shaohan Huang Li Dong Furu Wei* \\ Microsoft Research \\ \{wenwan, t-habao, shaohanh, lidongl, fuwei\}@microsoft.com
}

\begin{abstract}
We generalize deep self-attention distillation in MiniLM (Wang et al., 2020) by only using self-attention relation distillation for taskagnostic compression of pretrained Transformers. In particular, we define multi-head selfattention relations as scaled dot-product between the pairs of query, key, and value vectors within each self-attention module. Then we employ the above relational knowledge to train the student model. Besides its simplicity and unified principle, more favorably, there is no restriction in terms of the number of student's attention heads, while most previous work has to guarantee the same head number between teacher and student. Moreover, the fine-grained self-attention relations tend to fully exploit the interaction knowledge learned by Transformer. In addition, we thoroughly examine the layer selection strategy for teacher models, rather than just relying on the last layer as in MiNILM. We conduct extensive experiments on compressing both monolingual and multilingual pre-trained models. Experimental results demonstrate that our models ${ }^{1}$ distilled from base-size and large-size teachers (BERT, RoBERTa and XLM-R) outperform the state-of-the-art.
\end{abstract}

\section{Introduction}

Pretrained Transformers (Radford et al., 2018; Devlin et al., 2018; Dong et al., 2019; Yang et al., 2019; Joshi et al., 2019; Liu et al., 2019; Bao et al., 2020; Radford et al., 2019; Raffel et al., 2019; Lewis et al., 2019a) have been highly successful for a wide range of natural language processing tasks. However, these models usually consist of hundreds of millions of parameters and are getting bigger. It brings challenges for fine-tuning and online serv-

\footnotetext{
${ }^{*}$ Contact person.

${ }^{1}$ Distilled models and code will be publicly available at https://aka.ms/minilm.
}

ing in real-life applications due to the restrictions of computation resources and latency.

Knowledge distillation (KD; Hinton et al. 2015, Romero et al. 2015) has been widely employed to compress pretrained Transformers, which transfers knowledge of the large model (teacher) to the small model (student) by minimizing the differences between teacher and student features. Soft target probabilities (soft labels) and intermediate representations are usually utilized to perform KD training. In this work, we focus on task-agnostic compression of pretrained Transformers (Sanh et al., 2019; Tsai et al., 2019; Jiao et al., 2019; Sun et al., 2019b; Wang et al., 2020). The student models are distilled from large pretrained Transformers using large-scale text corpora. The distilled task-agnostic model can be directly finetuned on downstream tasks, and can be utilized to initialize task-specific distillation.

DistilBERT (Sanh et al., 2019) uses soft target probabilities for masked language modeling predictions and embedding outputs to train the student. The student model is initialized from the teacher by taking one layer out of two. TinyBERT (Jiao et al., 2019) utilizes hidden states and self-attention distributions (i.e., attention maps and weights), and adopts a uniform function to map student and teacher layers for layer-wise distillation. MobileBERT (Sun et al., 2019b) introduces specially designed teacher and student models using inverted-bottleneck and bottleneck structures to keep their layer number and hidden size the same, layer-wisely transferring hidden states and self-attention distributions. MINILM (Wang et al., 2020) proposes deep self-attention distillation, which uses self-attention distributions and value relations to help the student to deeply mimic teacher's self-attention modules. MiNILM shows that transferring knowledge of teacher's last layer achieves better performance than layer-wise distil- 
lation. In summary, most previous work relies on self-attention distributions to perform KD training, which leads to a restriction that the number of attention heads of student model has to be the same as its teacher.

In this work, we generalize and simplify deep self-attention distillation of MINILM (Wang et al., 2020) by using self-attention relation distillation. We introduce multi-head self-attention relations computed by scaled dot-product of pairs of queries, keys and values, which guides the student training. Taking query vectors as an example, in order to obtain queries of multiple relation heads, we first concatenate query vectors of different attention heads, and then split the concatenated vector according to the desired number of relation heads. Afterwards, for teacher and student models with different attention head numbers, we can align their queries with the same number of relation heads for distillation. Moreover, using a larger number of relation heads brings more fine-grained self-attention knowledge, which helps the student to achieves a deeper mimicry of teacher's self-attention module. In addition, for large-size (24 layers, 1024 hidden size) teachers, extensive experiments indicate that transferring an upper middle layer tends to perform better than using the last layer as in MiNILM.

Experimental results show that our monolingual models distilled from BERT and RoBERTa, and multilingual models distilled from XLM-R outperform state-of-the-art models in different parameter sizes. The $6 \times 768$ ( 6 layers, 768 hidden size) model distilled from BERT LARGE $_{\text {is }} 2.0 \times$ faster, meanwhile, performing better than BERT $\mathrm{BASE}_{\mathrm{S}}$. The base-size model distilled from RoBERTa LARGE $_{\text {out- }}$ performs RoBERTa $\mathrm{BASE}_{\mathrm{B} \text {. }}$ using much fewer training examples.

To summarize, our contributions include:

- We generalize and simplify deep self-attention distillation in MINILM by introducing multihead self-attention relation distillation, which brings more fine-grained self-attention knowledge and allows more flexibility for the number of student's attention heads.

- We conduct extensive distillation experiments on different large-size teachers and find that using knowledge of a teacher's upper middle layer achieves better performance.

- Experimental results demonstrate the effectiveness of our method for different monolin- gual and multilingual teachers in base-size and large-size.

\section{Related Work}

\subsection{Backbone Network: Transformer}

Multi-layer Transformer (Vaswani et al., 2017) has been widely adopted in pretrained models. Each Transformer layer consists of a self-attention sublayer and a position-wise fully connected feedforward sub-layer.

Self-Attention Transformer relies on multi-head self-attention to capture dependencies between words. Given previous Transformer layer's output $\mathbf{H}^{l-1} \in \mathbb{R}^{|x| \times d_{h}}$, the output of a self-attention head $\mathbf{O}_{l, a}, a \in\left[1, A_{h}\right]$ is computed via:

$$
\begin{gathered}
\mathbf{Q}_{l, a}=\mathbf{H}^{l-1} \mathbf{W}_{l, a}^{Q} \\
\mathbf{K}_{l, a}=\mathbf{H}^{l-1} \mathbf{W}_{l, a}^{K} \\
\mathbf{V}_{l, a}=\mathbf{H}^{l-1} \mathbf{W}_{l, a}^{V} \\
\mathbf{O}_{l, a}=\operatorname{softmax}\left(\frac{\mathbf{Q}_{l, a} \mathbf{K}_{l, a}^{\top}}{\sqrt{d_{k}}}\right) \mathbf{V}_{l, a}
\end{gathered}
$$

Previous layer's output is linearly projected to queries, keys and values using parameter matrices $\mathbf{W}_{l, a}^{Q}, \mathbf{W}_{l, a}^{K}, \mathbf{W}_{l, a}^{V} \in \mathbb{R}^{d_{h} \times d_{k}}$, respectively. The self-attention distributions are computed via scaled dot-product of queries and keys. These weights are assigned to the corresponding value vectors to obtain the attention output. $|x|$ represents the length of input sequence. $A_{h}$ and $d_{h}$ indicate the number of attention heads and hidden size. $d_{k}$ is the attention head size. $d_{k} \times A_{h}$ is usually equal to $d_{h}$.

\subsection{Pretrained Language Models}

Pre-training has led to strong improvements across a variety of natural language processing tasks. Pretrained language models are learned on large amounts of text data, and then fine-tuned to adapt to specific tasks. BERT (Devlin et al., 2018) proposes to pretrain a deep bidirectional Transformer using masked language modeling (MLM) objective. UNILM (Dong et al., 2019) is jointly pretrained on three types language modeling objectives to adapt to both understanding and generation tasks. RoBERTa (Liu et al., 2019) achieves strong performance by training longer steps using large batch size and more text data. MASS (Song et al., 2019), T5 (Raffel et al., 2019) and BART (Lewis et al., 


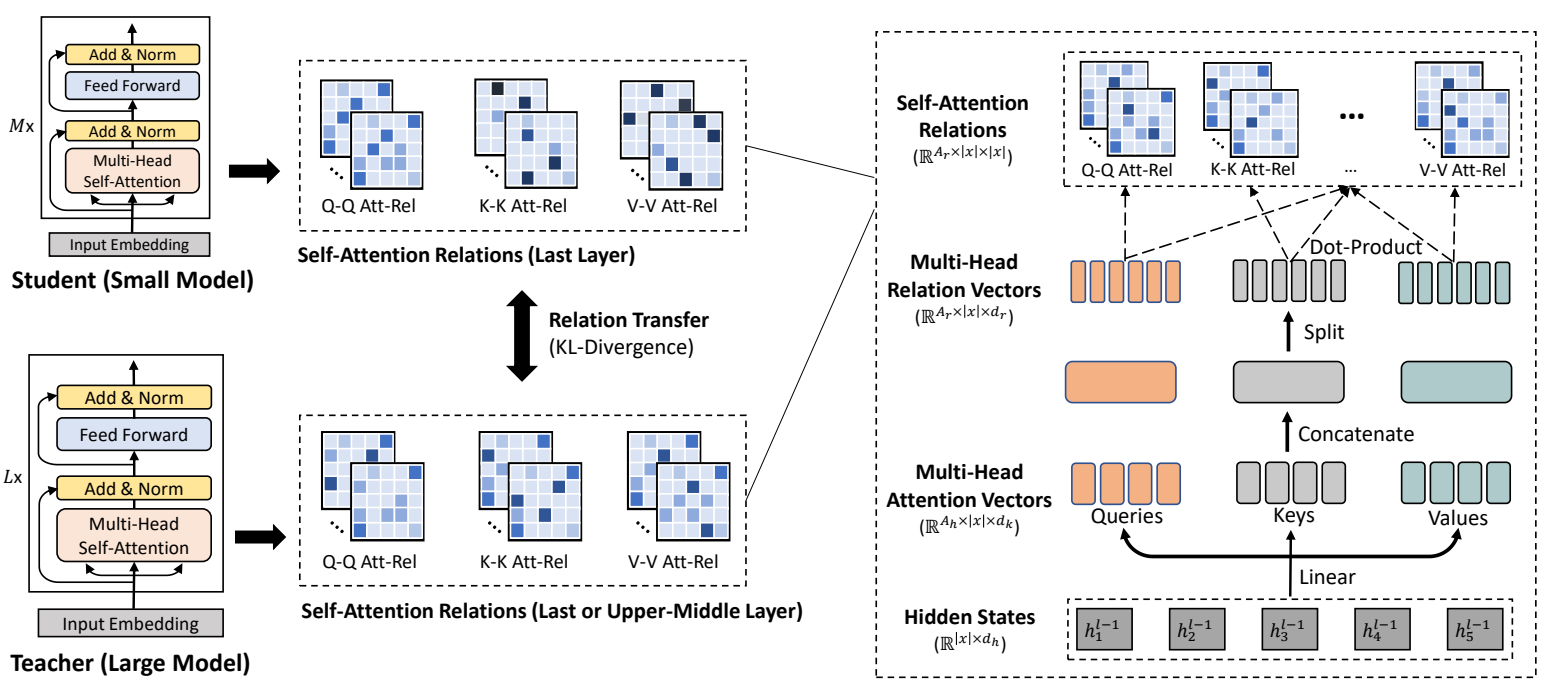

Figure 1: Overview of multi-head self-attention relation distillation. We introduce multi-head self-attention relations computed by scaled dot-product of pairs of queries, keys and values to guide the training of students. In order to obtain vectors (queries, keys and values) of multiple relation heads, we first concatenate self-attention vectors of different attention heads and then split them according to the desired number of relation heads. We choose to transfer Q-Q, K-K and V-V self-attention relations to achieve a balance between performance and training speed. For large-size teacher, we transfer the self-attention knowledge of an upper middle layer of the teacher. For base-size teacher, using the last layer achieves better performance.

2019a) employ a standard encoder-decoder structure and pretrain the decoder auto-regressively. Besides monolingual pretrained models, multilingual pretrained models (Devlin et al., 2018; Lample and Conneau, 2019; Chi et al., 2019; Conneau et al., 2019; Chi et al., 2020) also advance the state-of-theart on cross-lingual understanding and generation.

\subsection{Knowledge Distillation}

Knowledge distillation has been proven to be a promising way to compress large models while maintaining accuracy. Knowledge of a single or an ensemble of large models is used to guide the training of small models. Hinton et al. (2015) propose to use soft target probabilities to train student models. More fine-grained knowledge such as hidden states (Romero et al., 2015) and attention distributions (Zagoruyko and Komodakis, 2017; Hu et al., 2018) are introduced to improve the student model.

In this work, we focus on task-agnostic knowledge distillation of pretrained Transformers. The distilled task-agnostic model can be fine-tuned to adapt to downstream tasks. It can also be utilized to initialize task-specific distillation (Sun et al., 2019a; Turc et al., 2019; Aguilar et al., 2019; Mukherjee and Awadallah, 2020; Xu et al., 2020; Hou et al., 2020; Li et al., 2020), which uses a finetuned teacher model to guide the training of the student on specific tasks. Knowledge used for dis- tillation and layer mapping function are two key points for task-agnostic distillation of pretrained Transformers. Most previous work uses soft target probabilities, hidden states, self-attention distributions and value-relation to train the student model. For the layer mapping function, TinyBERT (Jiao et al., 2019) uses a uniform strategy to map teacher and student layers. MobileBERT (Sun et al., 2019b) assumes the student has the same number of layers as its teacher to perform layer-wise distillation. MiniLM (Wang et al., 2020) transfers selfattention knowledge of teacher's last layer to the student last Transformer layer. Different from previous work, our method uses multi-head selfattention relations to eliminate the restriction on the number of student's attention heads. Moreover, we show that transferring the self-attention knowledge of an upper middle layer of the large-size teacher model is more effective.

\section{Multi-Head Self-Attention Relation Distillation}

Following MiniLM (Wang et al., 2020), the key idea of our approach is to deeply mimic teacher's self-attention module, which draws dependencies between words and is the vital component of Transformer. MiNILM uses teacher's self-attention distributions to train the student model. It brings 


\begin{tabular}{|c|c|c|c|c|c|c|c|c|c|c|c|c|}
\hline Model & Teacher & \#Param & Speedup & SQuAD2 & MNLI-m & QNLI & QQP & RTE & SST & MRPC & CoLA & Avg \\
\hline BERT $_{\text {BASE }}$ & - & $109 \mathrm{M}$ & $\times 1.0$ & 76.8 & 84.5 & 91.7 & 91.3 & 68.6 & 93.2 & 87.3 & 58.9 & 81.5 \\
\hline RoBERTa $_{\text {BASE }}$ & - & $125 \mathrm{M}$ & $\times 1.0$ & 83.7 & 87.6 & 92.8 & 91.9 & 78.7 & 94.8 & 90.2 & 63.6 & 85.4 \\
\hline $\mathrm{BERT}_{\text {SMALL }}$ & - & $66 \mathrm{M}$ & $\times 2.0$ & 73.2 & 81.8 & 89.8 & 90.6 & 67.9 & 91.2 & 84.9 & 53.5 & 79.1 \\
\hline Truncated BERT $_{\mathrm{BASE}}$ & - & $66 \mathrm{M}$ & $\times 2.0$ & 69.9 & 81.2 & 87.9 & 90.4 & 65.5 & 90.8 & 82.7 & 41.4 & 76.2 \\
\hline Truncated RoBERTa $a_{\text {BASE }}$ & - & $81 \mathrm{M}$ & $\times 2.0$ & 77.9 & 84.9 & 91.1 & 91.3 & 67.9 & 92.9 & 87.5 & 55.2 & 81.1 \\
\hline DistilBERT & BERT $_{\text {BASE }}$ & $66 \mathrm{M}$ & $\times 2.0$ & 70.7 & 82.2 & 89.2 & 88.5 & 59.9 & 91.3 & 87.5 & 51.3 & 77.6 \\
\hline TinyBERT & BERT $_{\text {BASE }}$ & $66 \mathrm{M}$ & $\times 2.0$ & 73.1 & 83.5 & 90.5 & 90.6 & 72.2 & 91.6 & 88.4 & 42.8 & 79.1 \\
\hline MINILM & BERT $_{\text {BASE }}$ & $66 \mathrm{M}$ & $\times 2.0$ & 76.4 & 84.0 & 91.0 & 91.0 & 71.5 & 92.0 & 88.4 & 49.2 & 80.4 \\
\hline $6 \times 384$ Ours & BERT $_{\text {BASE }}$ & $22 \mathrm{M}$ & $\times 5.3$ & 72.9 & 82.8 & 90.3 & 90.6 & 68.9 & 91.3 & 86.6 & 41.8 & 78.2 \\
\hline $6 \times 384$ Ours & BERT $_{\text {LARGE }}$ & $22 \mathrm{M}$ & $\times 5.3$ & 74.3 & 83.0 & 90.4 & 90.7 & 68.5 & 91.1 & 87.8 & 41.6 & 78.4 \\
\hline $6 \times 384$ Ours & RoBERTa $_{\text {LARGE }}$ & $30 \mathrm{M}$ & $\times 5.3$ & 76.4 & 84.4 & 90.9 & 90.8 & 69.9 & 92.0 & 88.7 & 42.6 & 79.5 \\
\hline $6 \times 768$ Ours & BERT $_{\text {BASE }}$ & $66 \mathrm{M}$ & $\times 2.0$ & 76.3 & 84.2 & 90.8 & 91.1 & 72.1 & 92.4 & 88.9 & 52.5 & 81.0 \\
\hline $6 \times 768$ Ours & BERT $_{\text {LARGE }}$ & $66 \mathrm{M}$ & $\times 2.0$ & 77.7 & 85.0 & 91.4 & 91.1 & 73.0 & 92.5 & 88.9 & 53.9 & 81.7 \\
\hline $6 \times 768$ Ours & RoBERTa $_{\text {LARGE }}$ & $81 \mathrm{M}$ & $\times 2.0$ & 81.6 & 87.0 & 92.7 & 91.4 & 78.7 & 94.5 & 90.4 & 54.0 & 83.8 \\
\hline
\end{tabular}

Table 1: Results of our students distilled from base-size and large-size teachers on the development sets of GLUE and SQuAD 2.0. We report F1 for SQuAD 2.0, Matthews correlation coefficient for CoLA, and accuracy for other datasets. The GLUE results of DistilBERT are taken from Sanh et al. (2019). The rest results of DistilBERT, TinyBERT $^{2}$, BERT $_{\text {SMALL }}$, Truncated BERT BASE $_{\text {and MINILM are taken from Wang et al. (2020). BERT }}$ SMALL (Turc et al., 2019) is trained using the MLM objective, without using KD. We also report the results of truncated BERT $_{\mathrm{BASE}}$ and truncated RoBERTa $\mathrm{BASE}$, which drops the top 6 layers of the base model. Top-layer dropping has been proven to be a strong baseline (Sajjad et al., 2020). The fine-tuning results are an average of 4 runs.

the restriction on the number of attention heads of students, which is required to be the same as its teacher. To introduce more fine-grained selfattention knowledge and avoid using teacher's self-attention distributions, we generalize deep self-attention distillation in MINILM and introduce multi-head self-attention relations of pairs of queries, keys and values to train the student. Besides, we conduct extensive experiments and find that layer selection of the teacher model is critical for distilling large-size models. Figure 1 gives an overview of our method.

\subsection{Multi-Head Self-Attention Relations}

Multi-head self-attention relations are obtained by scaled dot-product of pairs ${ }^{3}$ of queries, keys and values of multiple relation heads. Taking query vectors as an example, in order to obtain queries of multiple relation heads, we first concatenate queries of different attention heads and then split the concatenated vector based on the desired number of relation heads. The same operation is also performed on keys and values. For teacher and student models which uses different number of attention heads, we convert their queries, keys and values of different number of attention heads into

\footnotetext{
${ }^{2}$ In addition to task-agnostic distillation, TinyBERT uses task-specific distillation and data augmentation to further improve the model. We report the fine-tuning results of their public task-agnostic model.

${ }^{3}$ There are nine types of self-attention relations, such as query-query, key-key, key-value and query-value relations.
}

vectors of the same number of relation heads to perform KD training. Our method eliminates the restriction on the number of attention heads of student models. Moreover, using more relation heads in computing self-attention relations brings more fine-grained self-attention knowledge and improves the performance of the student model.

We use $\mathbf{A}_{1}, \mathbf{A}_{2}, \mathbf{A}_{3}$ to denote the queries, keys and values of multiple relation heads. The KLdivergence between multi-head self-attention relations of the teacher and student is used as the training objective:

$$
\begin{gathered}
\mathcal{L}=\sum_{i=1}^{3} \sum_{j=1}^{3} \alpha_{i j} \mathcal{L}_{i j} \\
\mathcal{L}_{i j}=\frac{1}{A_{r}|x|} \sum_{a=1}^{A_{r}} \sum_{t=1}^{|x|} D_{K L}\left(\mathbf{R}_{i j, l, a, t}^{T} \| \mathbf{R}_{i j, m, a, t}^{S}\right) \\
\mathbf{R}_{i j, l, a}^{T}=\operatorname{softmax}\left(\frac{\mathbf{A}_{i, l, a}^{T} \mathbf{A}_{j, l, a}^{T_{\top}}}{\sqrt{d_{r}}}\right) \\
\mathbf{R}_{i j, m, a}^{S}=\operatorname{softmax}\left(\frac{\mathbf{A}_{i, m, a}^{S} \mathbf{A}_{j, m, a}^{S \top}}{\sqrt{d_{r}^{\prime}}}\right)
\end{gathered}
$$

where $\mathbf{A}_{i, l, a}^{T} \in \mathbb{R}^{|x| \times d_{r}}$ and $\mathbf{A}_{i, m, a}^{S} \in \mathbb{R}^{|x| \times d_{r}^{\prime}}(i \in$ $[1,3])$ are the queries, keys and values of a relation head of $l$-th teacher layer and $m$-th student layer. $d_{r}$ and $d_{r}^{\prime}$ are the relation head size of teacher and student models. $\mathbf{R}_{i j, l}^{T} \in \mathbb{R}^{A_{r} \times|x| \times|x|}$ is the selfattention relation of $\mathbf{A}_{i, l}^{T}$ and $\mathbf{A}_{j, l}^{T}$ of teacher model. 


\begin{tabular}{|c|c|c|c|c|c|c|c|c|c|c|c|c|c|}
\hline Model & Teacher & \#Param & Speedup & SQuAD2 & MNLI-m/mm & QNLI & QQP & RTE & SST & MRPC & CoLA & STS & Avg \\
\hline BERT $_{\text {BASE }}$ & - & $109 \mathrm{M}$ & $1.0 \times$ & 76.8 & $84.6 / 83.4$ & 90.5 & 71.2 & 66.4 & 93.5 & 88.9 & 52.1 & 85.8 & 79.3 \\
\hline BERT $_{\text {LARGE }}$ & - & $340 \mathrm{M}$ & $0.3 \times$ & 81.9 & $86.7 / 85.9$ & 92.7 & 72.1 & 70.1 & 94.9 & 89.3 & 60.5 & 86.5 & 82.1 \\
\hline $6 \times 768$ Ours & BERT $_{\text {BASE }}$ & $66 \mathrm{M}$ & $2.0 \times$ & 76.3 & $83.8 / 83.3$ & 90.2 & 70.9 & 69.2 & 92.9 & 89.1 & 46.6 & 84.3 & 78.7 \\
\hline $6 \times 768$ Ours & BERT $_{\text {LARGE }}$ & $66 \mathrm{M}$ & $2.0 \times$ & 77.7 & $84.5 / 84.0$ & 91.5 & 71.3 & 69.2 & 93.0 & 89.1 & 48.6 & 85.1 & 79.4 \\
\hline
\end{tabular}

Table 2: Results of our $6 \times 768$ students distilled form BERT on GLUE test sets and SQuAD 2.0 dev set. The reported results are directly fine-tuned on downstream tasks. We report F1 for SQuAD 2.0, QQP and MRPC, Spearman correlation for STS-B, Matthews correlation coefficient for CoLA and accuracy for the rest.

$\mathbf{R}_{i j, l, a}^{T} \in \mathbb{R}^{|x| \times|x|}$ is the self-attention relation of a teacher's relation head. $\mathbf{R}_{i j, m}^{S} \in \mathbb{R}^{A_{r} \times|x| \times|x|}$ is the self-attention relation of student model. For example, $\mathbf{R}_{11, l}^{T}$ represents teacher's Q-Q attention relation in Figure 1. $A_{r}$ is the number of relation heads. If the number of relation heads and attention heads is the same, the $\mathrm{Q}-\mathrm{K}$ relation is equivalent to the attention weights in self-attention module. $\alpha_{i j} \in\{0,1\}$ is the weight assigned to each selfattention relation loss. We transfer query-query, key-key and value-value self-attention relations to balance the performance and training cost.

\subsection{Layer Selection of Teacher Model}

Besides the knowledge used for distillation, mapping function between teacher and student layers is another key factor. As in MINILM, we only transfer the self-attention knowledge of one of the teacher layers to the student last layer. Only distilling one layer of the teacher model is fast and effective. Different from previous work which usually conducts experiments on base-size teachers, we experiment with different large-size teachers and find that transferring self-attention knowledge of an upper middle layer performs better than using other layers. For BERT LARGE $_{\text {and }}$ BERT LARGE-WwM, $_{\text {, }}$ transferring the 21-th (start at one) layer achieves the best performance. For RoBERTa ${ }_{\text {LARGE }}$ and XLM-R of 19-th layer achieves better performance. For the base-size teacher, we also find that using teacher's last layer performs better.

\section{Experiments}

We conduct distillation experiments on different teacher models including BERT $_{\mathrm{BASE}}, \mathrm{BERT}_{\mathrm{LARGE}}$, BERT $_{\text {LARGE-wwM }}$, RoBERTa $a_{\text {BASE }}$, RoBERTa $_{\text {LARGE }}$, XLM-R BASE $_{\text {and XLM-R }}$ LARGE.

\subsection{Setup}

We use the uncased version for three BERT teacher models. For the pre-training data, we use English
Wikipedia and BookCorpus (Zhu et al., 2015). We train student models using 256 as the batch size and $6 e-4$ as the peak learning rate for 400,000 steps. We use linear warmup over the first 4,000 steps and linear decay. We use Adam (Kingma and $\mathrm{Ba}$, 2015) with $\beta_{1}=0.9, \beta_{2}=0.999$. The maximum sequence length is set to 512 . The dropout rate and weight decay are 0.1 and 0.01 . The number of attention heads is 12 for all student models. The number of relation heads is 48 and 64 for base-size and large-size teacher model, respectively. The student models are initialized randomly.

For models distilled from RoBERTa, we use similar pre-training datasets as in Liu et al. (2019). For the $12 \times 768$ student model, we use Adam with $\beta_{1}=0.9, \beta_{2}=0.98$. The rest hyper-parameters are the same as models distilled from BERT.

For multilingual student models distilled from XLM-R, we perform training using the same datasets as in Conneau et al. (2019) for 1, 000, 000 steps. We conduct distillation experiments using 8 V100 GPUs with mixed precision training.

\subsection{Downstream Tasks}

Following previous pre-training (Devlin et al., 2018; Liu et al., 2019; Conneau et al., 2019) and task-agnostic distillation (Sun et al., 2019b; Jiao et al., 2019) work, we evaluate the English student models on GLUE benchmark and extractive question answering. The multilingual models are evaluated on cross-lingual natural language inference and cross-lingual question answering.

GLUE General Language Understanding Evaluation (GLUE) benchmark (Wang et al., 2019) consists of two single-sentence classification tasks (SST-2 (Socher et al., 2013) and CoLA (Warstadt et al., 2018)), three similarity and paraphrase tasks (MRPC (Dolan and Brockett, 2005), STS-B (Cer et al., 2017) and QQP), and four inference tasks (MNLI (Williams et al., 2018), QNLI (Rajpurkar et al., 2016), RTE (Dagan et al., 2006; Bar-Haim et al., 2006; Giampiccolo et al., 2007; Bentivogli 


\begin{tabular}{|c|c|c|c|c|c|c|c|c|c|c|c|c|}
\hline Model & Teacher & \#Param & SQuAD2 & MNLI & QNLI & QQP & RTE & SST & MRPC & CoLA & STS & Avg \\
\hline BERT $_{\text {BASE }}$ & - & $109 \mathrm{M}$ & 76.8 & 84.5 & 91.7 & 91.3 & 68.6 & 93.2 & 87.3 & 58.9 & 89.5 & 82.4 \\
\hline RoBERTa $_{\text {BASE }}$ & - & $125 \mathrm{M}$ & 83.7 & 87.6 & 92.8 & 91.9 & 78.7 & 94.8 & 90.2 & 63.6 & 91.2 & 86.1 \\
\hline $12 \times 768$ Ours & BERT $_{\text {LARGE }}$ & $109 \mathrm{M}$ & 81.8 & 86.5 & 92.6 & 91.6 & 76.4 & 93.3 & 89.2 & 62.3 & 90.5 & 84.9 \\
\hline $12 \times 768$ Ours & RoBERTa $_{\text {LARGE }}$ & $125 \mathrm{M}$ & 86.6 & 89.4 & 94.0 & 91.8 & 83.1 & 95.9 & 91.2 & 65.0 & 91.3 & 87.6 \\
\hline
\end{tabular}

Table 3: Results of our $12 \times 768$ models on the dev sets of GLUE benchmark and SQuAD 2.0. The fine-tuning results are an average of 4 runs for each task. We report F1 for SQuAD 2.0, Pearson correlation for STS-B, Matthews correlation coefficient for CoLA and accuracy for the rest.

\begin{tabular}{l|ccc|c}
\hline Model & SQuAD2 & MNLI-m & SST-2 & Avg \\
\hline MinILM (Last Layer) & 79.1 & 84.7 & 91.2 & 85.0 \\
$\quad$ Upper Middle Layer & 80.3 & 85.2 & 91.5 & 85.7 \\
\hline $12 \times 384$ Ours & $\mathbf{8 0 . 7}$ & $\mathbf{8 5 . 7}$ & $\mathbf{9 2 . 3}$ & $\mathbf{8 6 . 2}$ \\
\hline
\end{tabular}

Table 4: Comparison of different methods using BERT $_{\text {LARGE-WwM }}$ as the teacher. We report dev results of $12 \times 384$ student model with 128 embedding size.

et al., 2009) and WNLI (Levesque et al., 2012)).

Extractive Question Answering The task aims to predict a continuous sub-span of the passage to answer the question. We evaluate on SQuAD 2.0 (Rajpurkar et al., 2018), which has been served as a major question answering benchmark.

\section{Cross-lingual Natural Language Inference} (XNLI) XNLI (Conneau et al., 2018) is a cross-lingual classification benchmark. It aims to identity the semantic relationship between two sentences and provides instances in 15 languages.

Cross-lingual Question Answering We use MLQA (Lewis et al., 2019b) to evaluate multilingual models. MLQA extends English SQuAD dataset (Rajpurkar et al., 2016) to seven languages.

\subsection{Main Results}

Table 1 presents the dev results of $6 \times 384$ and $6 \times 768$ models distilled from BERT $\mathrm{BASE}_{\mathrm{B}}$, BERT $_{\text {LARGE }}$ and RoBERTa ${ }_{\text {LARGE }}$ on GLUE and SQuAD 2.0. (1) Previous methods (Sanh et al., 2019; Jiao et al., 2019; Sun et al., 2019a; Wang et al., 2020) usually distill BERT BASE $_{\text {into a } 6 \text {-layer }}$ model with 768 hidden size. We first report results of the same setting. Our $6 \times 768$ model outperforms DistilBERT, TinyBERT, MINILM and two BERT baselines across most tasks. Moreover, our method allows more flexibility for the number of attention heads of student models. (2) Both $6 \times 384$ and $6 \times 768$ models distilled from BERT LARGE $_{\text {out- }}$ perform models distilled from BERT BASE. The $_{\text {B }}$ $6 \times 768$ model distilled from BERT LARGE $_{\text {is }} 2.0 \times$ faster than $\mathrm{BERT}_{\mathrm{BASE}}$, while achieving better performance. (3) Student models distilled from RoBERTa $_{\text {LARGE }}$ achieve further improvements. Better teacher results in better students. Multi-head self-attention relation distillation is effective for different large-size pretrained Transformers.

We report the results of $6 \times 768$ students distilled from BERT BASE $_{\text {and }}$ BERT $_{\text {LARGE }}$ on GLUE test sets and SQuAD $2.0 \mathrm{dev}$ set in Table 2. $6 \times 768$ model distilled from BERT $_{\mathrm{BASE}}$ retains more than $99 \%$ accuracy of its teacher while using $50 \%$ Transformer parameters. $6 \times 768$ model distilled from BERT $_{\text {LARGE }}$ compares favorably with BERT BASE. $_{\text {. }}$

We compress RoBERTa ${ }_{\text {LARGE }}$ and BERT LARGE $_{\text {LAR }}$ into a base-size student model. Dev results of GLUE benchmark and SQuAD 2.0 are shown in Table 3. Our base-size models distilled from large-size teacher outperforms BERT $_{\mathrm{BASE}}$ and RoBERTa $_{\text {BASE }}$. Our method can be adopted to train students in different parameter size. Moreover, our student distilled from RoBERTa LARGE $_{\text {uses a much }}$ smaller (almost $32 \times$ smaller) training batch size and fewer training steps than RoBERTa ${ }_{\text {BASE. Our }}$ method requires much fewer training examples.

Most of previous work conducts experiments using base-size teachers. To compare with previous methods on large-size teacher, we reimplement

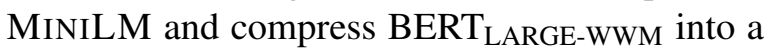
$12 \times 384$ student model. Dev results of SQuAD 2.0, MNLI-m and SST-2 are presented in Table 4. Our method also outperforms MINILM for large-size teachers. Moreover, we report results of distilling an upper middle layer instead of the last layer for MINILM. Layer selection is also effective for MinILM when distilling large-size teachers.

Table 5 and Table 6 show the results of our student models distilled from XLM-R on XNLI and MLQA. For XNLI, the best single model is selected on the joint dev set of all the languages as in Conneau et al. (2019). Following Lewis et al. (2019b), we adopt SQuAD 1.1 as training data and evaluate on MLQA English development set for 


\begin{tabular}{|c|c|c|c|c|c|c|c|c|c|c|c|c|c|c|c|c|c|c|c|c|}
\hline Model & Teacher & $\# \mathbf{L}$ & $\# \mathbf{H}$ & \#Param & en & fr & es & de & el & bg & ru & $\operatorname{tr}$ & ar & vi & th & $\mathbf{z h}$ & hi & sw & ur & Avg \\
\hline & - & 12 & 768 & $170 \mathrm{M}$ & 82.1 & 73.8 & 74.3 & 71.1 & 66.4 & 68.9 & 69.0 & 61.6 & 64.9 & 69.5 & 55.8 & 69.3 & 60.0 & 50.4 & 58.0 & 66.3 \\
\hline XLN & - & 16 & 1280 & $\mathrm{M}$ & 3.2 & 76.7 & 77.7 & 74.0 & 72.7 & 74.1 & 72.7 & 8.7 & 8.6 & 72.9 & 68.9 & 72.5 & 5.6 & 58.2 & 62.4 & 70.7 \\
\hline$R_{\text {BASE }}$ & - & 12 & 768 & $277 \mathrm{M}$ & 85.8 & 79.7 & 80.7 & 78.7 & 77.5 & 79.6 & 78.1 & 74.2 & 73.8 & 76.5 & 74.6 & 76.7 & 72.4 & 66.5 & 68.3 & 76.2 \\
\hline MINILM & XLM-R $\mathrm{BASE}$ & 6 & 384 & $107 \mathrm{M}$ & 79.2 & 72.3 & 73.1 & 70.3 & 69.1 & 72.0 & 69.1 & 64.5 & 64.9 & 69.0 & 66.0 & 67.8 & 62.9 & 59.0 & 60.6 & 68.0 \\
\hline & $\mathrm{XI}$ & 6 & 384 & & 78.1 & 71.4 & 72.7 & 69.2 & 70.8 & 72.2 & 69.9 & 67.5 & 66.1 & 68.9 & 67.5 & 68.2 & 64.7 & 62.7 & 62.3 & 68.8 \\
\hline $6 \times 384$ Ours & XLM-R & 6 & 384 & $107 \mathrm{M}$ & 79.8 & 72.5 & 73.7 & 69.3 & 70.6 & 72.3 & 69.5 & 66.9 & 67.5 & 69.1 & 67.0 & 68.7 & 64.8 & 62.4 & 63.0 & 69.1 \\
\hline
\end{tabular}

Table 5: Cross-lingual classification results of our $6 \times 384$ multilingual models on XNLI. We report the accuracy on each of the $15 \mathrm{XNLI}$ languages and the average accuracy. \#L and \#H indicate the number of layers and hidden size.

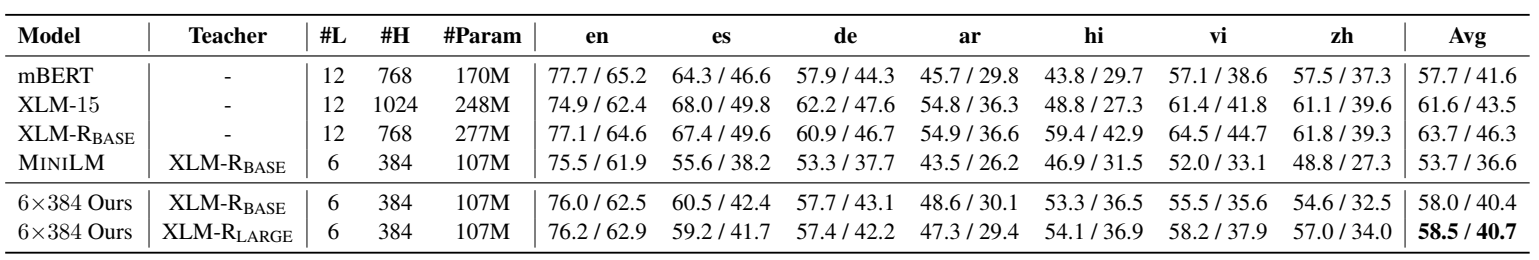

Table 6: Cross-lingual question answering results of our $6 \times 384$ multilingual models on MLQA. We report the F1 and EM (exact match) scores on each of the 7 MLQA languages. \#L and \#H indicate the number of layers and hidden size.

\begin{tabular}{l|ccc|c}
\hline Model & SQuAD2 & MNLI-m & SST-2 & Avg \\
\hline Ours (Q-Q + K-K + V-V) & $\mathbf{7 2 . 8}$ & $\mathbf{8 2 . 2}$ & $\mathbf{9 1 . 5}$ & $\mathbf{8 2 . 2}$ \\
- Q-Q Att-Rel & 71.6 & 81.9 & 90.6 & 81.4 \\
- K-K Att-Rel & 71.9 & 81.9 & 90.5 & 81.4 \\
- V-V Att-Rel & 71.5 & 81.6 & 90.5 & 81.2 \\
Q-K + V-V Att-Rels & 72.4 & $\mathbf{8 2 . 2}$ & 91.0 & 81.9 \\
\hline
\end{tabular}

Table 7: Ablation studies of different self-attention relations. We report results of $6 \times 384$ student model dis-

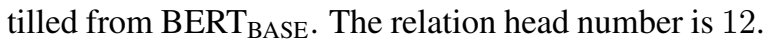

\begin{tabular}{lcccc}
\hline \#Relation Heads & 6 & 12 & 24 & $\mathbf{4 8}$ \\
\hline $6 \times 384$ model distilled from $R o B E R T a_{\text {BASE }}$ \\
MNLI-m & 82.8 & 82.9 & 83.0 & $\mathbf{8 3 . 4}$ \\
SQuAD 2.0 & 74.5 & 75.0 & 74.9 & $\mathbf{7 5 . 7}$ \\
\hline $6 \times 384$ model distilled from $B E R T_{B A S E}$ \\
MNLI-m & 81.9 & 82.2 & 82.2 & $\mathbf{8 2 . 4}$ \\
SQuAD 2.0 & 71.9 & 72.8 & 72.7 & $\mathbf{7 3 . 0}$ \\
\hline
\end{tabular}

Table 8: Results of $6 \times 384$ model using different number of relation heads.

early stopping. Our $6 \times 384$ model outperforms mBERT (Devlin et al., 2018) with $5.3 \times$ speedup. Our method also performs better than MINILM, which further validates the effectiveness of multihead self-attention relation distillation. Transferring multi-head self-attention relations can bring more fine-grained self-attention knowledge.

\subsection{Ablation Studies}

Effect of using different self-attention relations We perform ablation studies to analyse the contribution of different self-attention relations. Dev results of three tasks are illustrated in Table 7. Q$\mathrm{Q}, \mathrm{K}-\mathrm{K}$ and $\mathrm{V}-\mathrm{V}$ self-attention relations positively contribute to the final results. Besides, we also compare $\mathrm{Q}-\mathrm{Q}+\mathrm{K}-\mathrm{K}+\mathrm{V}-\mathrm{V}$ with $\mathrm{Q}-\mathrm{K}+\mathrm{V}-\mathrm{V}$ given queries and keys are employed to compute selfattention distributions in self-attention module. Experimental result shows that using Q-Q + K-K + $\mathrm{V}-\mathrm{V}$ achieves better performance.

Effect of distilling different teacher layers Figure 2 presents the results of $6 \times 384$ model distilled from different layers of BERT $\mathrm{BASE}_{\mathrm{B}}, \mathrm{BERT}_{\mathrm{LARGE}}$ and XLM-R LARGE$_{\text {. For BERT }}$ BASE, using the last layer achieves better performance than other layers. For BERT LARGE $_{\text {and XLM-R }}$ LARGE, we find that using one of the upper middle layers achieves the best performance. The same trend is also observed for BERT $_{\text {LARGE-WwM }}$ and RoBERTa LARGE $_{\text {. }}$.

Effect of different number of relation heads Table 8 shows the results of $6 \times 384$ model distilled from BERT BASE $_{\text {and RoBERTa }}$ BASE using different number of relation heads. Using a larger number of relation heads achieves better performance. More fine-grained self-attention knowledge can be captured by using more relation heads, which helps the student to deeply mimic the self-attention module of its teacher. Besides, we find that the number of relation heads is not required to be a positive multiple of both the number of student and teacher attention heads. The relation head can be a fragment of a single attention head or contains fragments from 


\begin{tabular}{|c|c|c|c|c|c|c|c|c|c|c|c|c|c|}
\hline Model & Teacher & \#Param & Speedup & SQuAD2 & MNLI-m/mm & QNLI & QQP & RTE & SST & MRPC & CoLA & STS & Avg \\
\hline BERT $_{\text {BASE }}$ & - & $109 \mathrm{M}$ & $1.0 \times$ & 76.8 & $84.6 / 83.4$ & 90.5 & 71.2 & 66.4 & 93.5 & 88.9 & 52.1 & 85.8 & 79.3 \\
\hline MobileBERT & IB-BERT $_{\text {LARGE }}$ & $25 \mathrm{M}$ & $1.8 \times$ & 80.2 & $84.3 / 83.4$ & 91.6 & 70.5 & 70.4 & 92.6 & 88.8 & 51.1 & 84.8 & 79.8 \\
\hline + More Att-Rels & BERT LARGE-WWM $_{\text {LW }}$ & $25 \mathrm{M}$ & $2.7 \times$ & 80.9 & $85.8 / 84.8$ & 92.3 & 71.6 & 72.0 & 93.6 & 89.7 & 46.6 & 86.0 & 80.3 \\
\hline
\end{tabular}

Table 9: Comparison between MobileBERT and the same-size model (12 layers, 384 hidden size and 128 embed-

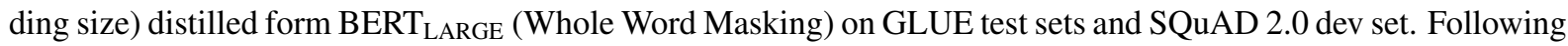
MobileBERT (Sun et al., 2019b), the reported results are directly fine-tuned on downstream tasks. We compute the speedup of MobileBERT according to their reported latency.

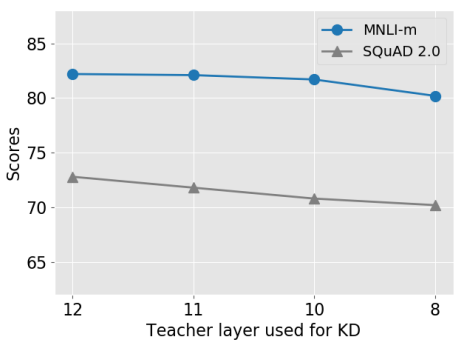

(a) BERT $_{\text {BASE }}$ as the teacher

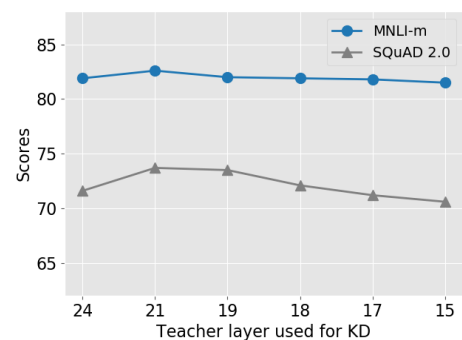

(b) BERT $_{\text {LARGE }}$ as the teacher

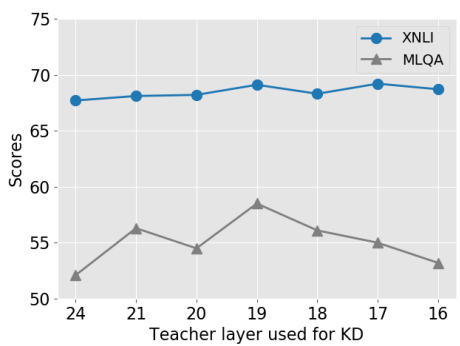

(c) XLM-R $\mathrm{LARGE}_{\text {as }}$ the teacher

Figure 2: $6 \times 384$ models trained using different $\operatorname{BERT}_{\mathrm{BASE}}(\mathrm{a}), \mathrm{BERT}_{\mathrm{LARGE}}(\mathrm{b})$ and XLM-R $\mathrm{LARGE}_{\text {(c) layers. }}$

\begin{tabular}{l|c|ccc}
\hline Model & Teacher & SQuAD2 & MNLI-m & SST-2 \\
\hline $6 \times 384$ Ours & BERT $_{\text {BASE }}$ & 72.9 & $\mathbf{8 2 . 8}$ & 91.3 \\
+ More Att-Rels & BERT $_{\text {BASE }}$ & $\mathbf{7 3 . 3}$ & $\mathbf{8 2 . 8}$ & $\mathbf{9 1 . 6}$ \\
\hline $6 \times 384$ Ours & BERT $_{\text {LARGE }}$ & 74.3 & 83.0 & 91.1 \\
+ More Att-Rels & BERT $_{\text {LARGE }}$ & $\mathbf{7 4 . 7}$ & $\mathbf{8 3 . 2}$ & $\mathbf{9 2 . 4}$ \\
\hline $6 \times 384$ Ours & RoBERTa $_{\text {LARGE }}$ & $\mathbf{7 6 . 4}$ & $\mathbf{8 4 . 4}$ & 92.0 \\
+ More Att-Rels & RoBERTa $_{\text {LARGE }}$ & 76.0 & $\mathbf{8 4 . 4}$ & $\mathbf{9 2 . 1}$ \\
\hline $6 \times 768$ Ours & BERT $_{\text {BASE }}$ & 76.3 & 84.2 & $\mathbf{9 2 . 4}$ \\
+ More Att-Rels & BERT $_{\text {BASE }}$ & $\mathbf{7 6 . 8}$ & $\mathbf{8 4 . 4}$ & 92.3 \\
\hline $6 \times 768$ Ours & BERT $_{\text {LARGE }}$ & 77.7 & 85.0 & $\mathbf{9 2 . 5}$ \\
+ More Att-Rels & BERT $_{\text {LARGE }}$ & $\mathbf{7 8 . 1}$ & $\mathbf{8 5 . 2}$ & $\mathbf{9 2 . 5}$ \\
\hline $6 \times 768$ Ours & RoBERTa $_{\text {LARGE }}$ & $\mathbf{8 1 . 6}$ & 87.0 & $\mathbf{9 4 . 5}$ \\
+ More Att-Rels & RoBERTa $_{\text {LARGE }}$ & 81.2 & $\mathbf{8 7 . 3}$ & 94.1 \\
\hline
\end{tabular}

Table 10: Results of introducing more self-attention relations (Q-K, K-Q, Q-V, V-Q, K-V and V-K relations).

multiple attention heads.

\section{Discussion}

\subsection{Comparison with MobileBERT}

MobileBERT (Sun et al., 2019b) compresses a specially designed teacher model (in the BERT $\mathrm{LARGE}_{\mathrm{L}}$ size) with inverted bottleneck modules into a 24layer student using the bottleneck modules. Since our goal is to compress different large models (e.g. BERT and RoBERTa) to small models using standard Transformer architecture, we note that our student model can not directly compare with MobileBERT. We provide results of a student model with the same parameter size for a reference. A public large-size model (BERT LARGE-WwM) is used as the teacher, which achieves similar performance as MobileBERT's teacher. We distill BERT LARGE-WwM into a student model (25M parameters) using the same training data (i.e., English Wikipedia and BookCorpus). The test results of GLUE and dev result of SQuAD 2.0 are illustrated in Table 9. Our model outperforms MobileBERT across most tasks with a faster inference speed. Moreover, our method can be applied for different teachers and has much fewer restriction of students.

We also observe that our model performs relatively worse on CoLA compared with MobileBERT. The task of CoLA is to evaluate the grammatical acceptability of a sentence. It requires more fine-grained linguistic knowledge that can be learnt from language modeling objectives. Fine-tuning the model using the MLM objective as in MobileBERT brings improvements for CoLA. However, our preliminary experiments show that this strategy will lead to slight drop for other GLUE tasks.

\subsection{Results of More Self-Attention Relations}

In Table 9 and 10, we report results of students trained using more self-attention relations $(\mathrm{Q}-\mathrm{K}, \mathrm{K}$ $\mathrm{Q}, \mathrm{Q}-\mathrm{V}, \mathrm{V}-\mathrm{Q}, \mathrm{K}-\mathrm{V}$ and V-K relations). We observe improvements across most tasks, especially for student models distilled from BERT. Fine-grained selfattention knowledge in more attention relations improves our students. However, introducing more self-attention relations also brings a higher compu- 
tational cost. In order to achieve a balance between performance and computational cost, we choose to transfer Q-Q, K-K and V-V self-attention relations instead of all self-attention relations in this work.

\section{Conclusion}

We generalize deep self-attention distillation in MINILM by employing multi-head self-attention relations to train the student. Our method introduces more fine-grained self-attention knowledge and eliminates the restriction of the number of student's attention heads. Moreover, we show that transferring the self-attention knowledge of an upper middle layer achieves better performance for large-size teachers. Our monolingual and multilingual models distilled from BERT, RoBERTa and XLM-R obtain competitive performance and outperform state-of-the-art methods. For future work, we are exploring an automatic layer selection algorithm. We also would like to apply our method to larger pretrained Transformers.

\section{References}

Gustavo Aguilar, Yuan Ling, Yu Zhang, Benjamin Yao, Xing Fan, and Edward Guo. 2019. Knowledge distillation from internal representations. CoRR, abs/1910.03723.

Hangbo Bao, Li Dong, Furu Wei, Wenhui Wang, Nan Yang, Xiaodong Liu, Yu Wang, Songhao Piao, Jianfeng Gao, Ming Zhou, and Hsiao-Wuen Hon. 2020. Unilmv2: Pseudo-masked language models for unified language model pre-training. arXiv preprint arXiv:2002.12804.

Roy Bar-Haim, Ido Dagan, Bill Dolan, Lisa Ferro, and Danilo Giampiccolo. 2006. The second PASCAL recognising textual entailment challenge. In Proceedings of the Second PASCAL Challenges Workshop on Recognising Textual Entailment.

Luisa Bentivogli, Ido Dagan, Hoa Trang Dang, Danilo Giampiccolo, and Bernardo Magnini. 2009. The fifth pascal recognizing textual entailment challenge. In In Proc Text Analysis Conference (TAC'09.

Daniel Cer, Mona Diab, Eneko Agirre, Inigo LopezGazpio, and Lucia Specia. 2017. Semeval-2017 task 1: Semantic textual similarity-multilingual and cross-lingual focused evaluation. arXiv preprint arXiv:1708.00055.

Zewen Chi, Li Dong, Furu Wei, Wenhui Wang, Xianling Mao, and Heyan Huang. 2019. Cross-lingual natural language generation via pre-training. CoRR, abs/1909.10481.
Zewen Chi, Li Dong, Furu Wei, Nan Yang, Saksham Singhal, Wenhui Wang, Xia Song, XianLing Mao, Heyan Huang, and Ming Zhou. 2020. Infoxlm: An information-theoretic framework for cross-lingual language model pre-training. CoRR, abs/2007.07834.

Alexis Conneau, Kartikay Khandelwal, Naman Goyal, Vishrav Chaudhary, Guillaume Wenzek, Francisco Guzmán, Edouard Grave, Myle Ott, Luke Zettlemoyer, and Veselin Stoyanov. 2019. Unsupervised cross-lingual representation learning at scale. CoRR, abs/1911.02116.

Alexis Conneau, Guillaume Lample, Ruty Rinott, Adina Williams, Samuel R Bowman, Holger Schwenk, and Veselin Stoyanov. 2018. Xnli: Evaluating crosslingual sentence representations. In Proceedings of the 2018 Conference on Empirical Methods in Natural Language Processing (EMNLP).

Ido Dagan, Oren Glickman, and Bernardo Magnini. 2006. The pascal recognising textual entailment challenge. In Proceedings of the First International Conference on Machine Learning Challenges: Evaluating Predictive Uncertainty Visual Object Classification, and Recognizing Textual Entailment, MLCW'05, pages 177-190, Berlin, Heidelberg. Springer-Verlag.

Jacob Devlin, Ming-Wei Chang, Kenton Lee, and Kristina Toutanova. 2018. BERT: pre-training of deep bidirectional transformers for language understanding. CoRR, abs/1810.04805.

William B Dolan and Chris Brockett. 2005. Automatically constructing a corpus of sentential paraphrases. In Proceedings of the Third International Workshop on Paraphrasing (IWP2005).

Li Dong, Nan Yang, Wenhui Wang, Furu Wei, Xiaodong Liu, Yu Wang, Jianfeng Gao, Ming Zhou, and Hsiao-Wuen Hon. 2019. Unified language model pre-training for natural language understanding and generation. In 33rd Conference on Neural Information Processing Systems (NeurIPS 2019).

Danilo Giampiccolo, Bernardo Magnini, Ido Dagan, and Bill Dolan. 2007. The third PASCAL recognizing textual entailment challenge. In Proceedings of the ACL-PASCAL Workshop on Textual Entailment and Paraphrasing, pages 1-9, Prague. Association for Computational Linguistics.

Geoffrey E. Hinton, Oriol Vinyals, and Jeffrey Dean. 2015. Distilling the knowledge in a neural network. CoRR, abs/1503.02531.

Lu Hou, Zhiqi Huang, Lifeng Shang, Xin Jiang, Xiao Chen, and Qun Liu. 2020. Dynabert: Dynamic BERT with adaptive width and depth. In Advances in Neural Information Processing Systems 33: Annual Conference on Neural Information Processing Systems 2020, NeurIPS 2020, December 6-12, 2020, virtual. 
Minghao Hu, Yuxing Peng, Furu Wei, Zhen Huang, Dongsheng Li, Nan Yang, and Ming Zhou. 2018 Attention-guided answer distillation for machine reading comprehension. In Proceedings of the 2018 Conference on Empirical Methods in Natural Language Processing, Brussels, Belgium, October 31 November 4, 2018, pages 2077-2086.

Xiaoqi Jiao, Yichun Yin, Lifeng Shang, Xin Jiang, Xiao Chen, Linlin Li, Fang Wang, and Qun Liu. 2019. Tinybert: Distilling BERT for natural language understanding. CoRR, abs/1909.10351.

Mandar Joshi, Danqi Chen, Yinhan Liu, Daniel S Weld, Luke Zettlemoyer, and Omer Levy. 2019. Spanbert: Improving pre-training by representing and predicting spans. arXiv preprint arXiv:1907.10529.

Diederik P. Kingma and Jimmy Ba. 2015. Adam: A method for stochastic optimization. In 3rd International Conference on Learning Representations, San Diego, CA.

Guillaume Lample and Alexis Conneau. 2019. Crosslingual language model pretraining. Advances in Neural Information Processing Systems (NeurIPS).

Hector Levesque, Ernest Davis, and Leora Morgenstern. 2012. The winograd schema challenge. In Thirteenth International Conference on the Principles of Knowledge Representation and Reasoning.

Mike Lewis, Yinhan Liu, Naman Goyal, Marjan Ghazvininejad, Abdelrahman Mohamed, Omer Levy, Veselin Stoyanov, and Luke Zettlemoyer. 2019a. BART: Denoising sequence-to-sequence pre-training for natural language generation, translation, and comprehension. arXiv preprint arXiv:1910.13461.

Patrick S. H. Lewis, Barlas Oguz, Ruty Rinott, Sebastian Riedel, and Holger Schwenk. 2019b. MLQA: evaluating cross-lingual extractive question answering. CoRR, abs/1910.07475.

Jianquan Li, Xiaokang Liu, Honghong Zhao, Ruifeng Xu, Min Yang, and Yaohong Jin. 2020. BERT-EMD: many-to-many layer mapping for BERT compression with earth mover's distance. In Proceedings of the 2020 Conference on Empirical Methods in Natural Language Processing, EMNLP 2020, Online, November 16-20, 2020, pages 3009-3018. Association for Computational Linguistics.

Yinhan Liu, Myle Ott, Naman Goyal, Jingfei Du, Mandar Joshi, Danqi Chen, Omer Levy, Mike Lewis, Luke Zettlemoyer, and Veselin Stoyanov. 2019. RoBERTa: A robustly optimized BERT pretraining approach. arXiv preprint arXiv:1907.11692.

Subhabrata Mukherjee and Ahmed Hassan Awadallah. 2020. Xtremedistil: Multi-stage distillation for massive multilingual models. In Proceedings of the 58th Annual Meeting of the Association for Computational Linguistics, ACL 2020, Online, July 5-10, 2020, pages 2221-2234. Association for Computational Linguistics.
Alec Radford, Karthik Narasimhan, Tim Salimans, and Ilya Sutskever. 2018. Improving language understanding by generative pre-training.

Alec Radford, Jeff Wu, Rewon Child, David Luan, Dario Amodei, and Ilya Sutskever. 2019. Language models are unsupervised multitask learners.

Colin Raffel, Noam Shazeer, Adam Roberts, Katherine Lee, Sharan Narang, Michael Matena, Yanqi Zhou, Wei Li, and Peter J. Liu. 2019. Exploring the limits of transfer learning with a unified text-to-text transformer. arXiv preprint arXiv:1910.10683.

Pranav Rajpurkar, Robin Jia, and Percy Liang. 2018. Know what you don't know: Unanswerable questions for SQuAD. In Proceedings of the 56th Annual Meeting of the Association for Computational Linguistics, ACL 2018, Melbourne, Australia, July 15-20, 2018, Volume 2: Short Papers, pages 784789.

Pranav Rajpurkar, Jian Zhang, Konstantin Lopyrev, and Percy Liang. 2016. SQuAD: 100,000+ questions for machine comprehension of text. In Proceedings of the 2016 Conference on Empirical Methods in Natural Language Processing, pages 2383-2392, Austin, Texas. Association for Computational Linguistics.

Adriana Romero, Nicolas Ballas, Samira Ebrahimi Kahou, Antoine Chassang, Carlo Gatta, and Yoshua Bengio. 2015. Fitnets: Hints for thin deep nets. In 3 rd International Conference on Learning Representations, ICLR 2015, San Diego, CA, USA, May 7-9, 2015, Conference Track Proceedings.

Hassan Sajjad, Fahim Dalvi, Nadir Durrani, and Preslav Nakov. 2020. Poor man's BERT: smaller and faster transformer models. CoRR, abs/2004.03844.

Victor Sanh, Lysandre Debut, Julien Chaumond, and Thomas Wolf. 2019. Distilbert, a distilled version of BERT: smaller, faster, cheaper and lighter. CoRR, abs/1910.01108.

Richard Socher, Alex Perelygin, Jean Wu, Jason Chuang, Christopher D Manning, Andrew Ng, and Christopher Potts. 2013. Recursive deep models for semantic compositionality over a sentiment treebank. In Proceedings of the 2013 conference on empirical methods in natural language processing, pages 1631-1642.

Kaitao Song, Xu Tan, Tao Qin, Jianfeng Lu, and TieYan Liu. 2019. Mass: Masked sequence to sequence pre-training for language generation. arXiv preprint arXiv:1905.02450.

Siqi Sun, Yu Cheng, Zhe Gan, and Jingjing Liu. 2019a. Patient knowledge distillation for BERT model compression. In Proceedings of the 2019 Conference on Empirical Methods in Natural Language Processing and the 9th International Joint Conference on Natural Language Processing, EMNLP-IJCNLP 2019, Hong Kong, China, November 3-7, 2019, pages 4322-4331. 
Zhiqing Sun, Hongkun Yu, Xiaodan Song, Renjie Liu, Yiming Yang, and Denny Zhou. 2019b. Mobilebert: Task-agnostic compression of bert by progressive knowledge transfer.

Henry Tsai, Jason Riesa, Melvin Johnson, Naveen Arivazhagan, Xin Li, and Amelia Archer. 2019. Small and practical BERT models for sequence labeling. In Proceedings of the 2019 Conference on Empirical Methods in Natural Language Processing and the 9th International Joint Conference on Natural Language Processing, EMNLP-IJCNLP 2019, Hong Kong, China, November 3-7, 2019, pages 36303634 .

Iulia Turc, Ming-Wei Chang, Kenton Lee, and Kristina Toutanova. 2019. Well-read students learn better: The impact of student initialization on knowledge distillation. CoRR, abs/1908.08962.

Ashish Vaswani, Noam Shazeer, Niki Parmar, Jakob Uszkoreit, Llion Jones, Aidan N Gomez, Łukasz Kaiser, and Illia Polosukhin. 2017. Attention is all you need. In Advances in Neural Information Processing Systems 30, pages 5998-6008. Curran Associates, Inc.

Alex Wang, Amanpreet Singh, Julian Michael, Felix Hill, Omer Levy, and Samuel R. Bowman. 2019. GLUE: A multi-task benchmark and analysis platform for natural language understanding. In International Conference on Learning Representations.

Wenhui Wang, Furu Wei, Li Dong, Hangbo Bao, Nan Yang, and Ming Zhou. 2020. Minilm: Deep selfattention distillation for task-agnostic compression of pre-trained transformers. In Advances in Neural Information Processing Systems 33: Annual Conference on Neural Information Processing Systems 2020, NeurIPS 2020, December 6-12, 2020, virtual.

Alex Warstadt, Amanpreet Singh, and Samuel R Bowman. 2018. Neural network acceptability judgments. arXiv preprint arXiv:1805.12471.

Adina Williams, Nikita Nangia, and Samuel Bowman. 2018. A broad-coverage challenge corpus for sentence understanding through inference. In Proceedings of the 2018 Conference of the North American Chapter of the Association for Computational Linguistics: Human Language Technologies, Volume 1 (Long Papers), pages 1112-1122, New Orleans, Louisiana. Association for Computational Linguistics.

Canwen Xu, Wangchunshu Zhou, Tao Ge, Furu Wei, and Ming Zhou. 2020. Bert-of-theseus: Compressing BERT by progressive module replacing. CoRR, abs/2002.02925.

Zhilin Yang, Zihang Dai, Yiming Yang, Jaime Carbonell, Ruslan Salakhutdinov, and Quoc V Le. 2019. XLNet: Generalized autoregressive pretraining for language understanding. In 33rd Conference on Neural Information Processing Systems (NeurIPS 2019).

\begin{tabular}{|c|c|c|c|c|}
\hline Corpus & \#Train & \#Dev & \#Test & Metrics \\
\hline \multicolumn{5}{|c|}{ Single-Sentence Tasks } \\
\hline CoLA & $8.5 \mathrm{k}$ & $1 \mathrm{k}$ & $1 \mathrm{k}$ & Matthews Corr \\
\hline SST-2 & $67 \mathrm{k}$ & 872 & $1.8 \mathrm{k}$ & Accuracy \\
\hline \multicolumn{5}{|c|}{ Similarity and Paraphrase Tasks } \\
\hline QQP & $364 \mathrm{k}$ & $40 \mathrm{k}$ & $391 \mathrm{k}$ & Accuracy/F1 \\
\hline MRPC & $3.7 \mathrm{k}$ & 408 & $1.7 \mathrm{k}$ & Accuracy/F1 \\
\hline STS-B & $7 \mathrm{k}$ & $1.5 \mathrm{k}$ & $1.4 \mathrm{k}$ & Pearson/Spearman Corr \\
\hline \multicolumn{5}{|c|}{ Inference Tasks } \\
\hline MNLI & $393 k$ & $20 \mathrm{k}$ & $20 \mathrm{k}$ & Accuracy \\
\hline RTE & $2.5 \mathrm{k}$ & 276 & $3 \mathrm{k}$ & Accuracy \\
\hline QNLI & $105 \mathrm{k}$ & $5.5 \mathrm{k}$ & $5.5 \mathrm{k}$ & Accuracy \\
\hline WNLI & 634 & 71 & 146 & Accuracy \\
\hline
\end{tabular}

Table 11: Summary of the GLUE benchmark.

\begin{tabular}{cccc}
\hline \#Train & \#Dev & \#Test & Metrics \\
\hline 130,319 & 11,873 & 8,862 & Exact Match/F1 \\
\hline
\end{tabular}

Table 12: Dataset statistics and metrics of SQuAD 2.0.

Sergey Zagoruyko and Nikos Komodakis. 2017. Paying more attention to attention: Improving the performance of convolutional neural networks via attention transfer. In 5th International Conference on Learning Representations, ICLR 2017, Toulon, France, April 24-26, 2017, Conference Track Proceedings.

Yukun Zhu, Ryan Kiros, Rich Zemel, Ruslan Salakhutdinov, Raquel Urtasun, Antonio Torralba, and Sanja Fidler. 2015. Aligning books and movies: Towards story-like visual explanations by watching movies and reading books. In Proceedings of the IEEE international conference on computer vision, pages 1927.

\section{A GLUE Benchmark}

The summary of datasets used for the General Language Understanding Evaluation (GLUE) benchmark $^{4}$ (Wang et al., 2019) is presented in Table 11.

\section{B SQUAD 2.0}

We present the dataset statistics and metrics of SQuAD 2.0 ${ }^{5}$ (Rajpurkar et al., 2018) in Table 12.

\section{Hyper-parameters for Fine-tuning}

Extractive Question Answering For SQuAD 2.0, the maximum sequence length is 384 . The batch size is set to 32 . We choose learning rates from $\{3 e-5,6 e-5,8 e-5,9 e-5\}$ and fine-tune the model for 3 epochs. The warmup ration and weight decay is 0.1 and 0.01 .

\footnotetext{
${ }^{4}$ https: / / gluebenchmark.com/

${ }^{5}$ http: //stanford-ga.com
} 
GLUE The maximum sequence length is 128 for the GLUE benchmark. We set batch size to 32 , choose learning rates from $\{1 \mathrm{e}-5,1.5 \mathrm{e}-5,2 \mathrm{e}-5,3 \mathrm{e}-$ $5,5 e-5\}$ and epochs from $\{3,5,10\}$ for different student models. We fine-tune CoLA task with longer training steps (25 epochs). The warmup ration and weight decay is 0.1 and 0.01 .

Cross-lingual Natural Language Inference (XNLI) The maximum sequence length is 128 for XNLI. We fine-tune 5 epochs using 128 as the batch size. The learning rates are chosen from $\{5 e-5,6 e-5\}$.

Cross-lingual Question Answering For MLQA, the maximum sequence length is 512 . We fine-tune 3 epochs using 32 as the batch size. The learning rates are chosen from $\{5 e-5,6 e-5\}$. 